\title{
Salter-Harris type II fracture of the middle phalanx with concomitant central slip injury in an 11 year old: a case report
}

\author{
Devan O. Higginbotham*, Andrew G. Tsai
}

Department of Orthopaedic Surgery, Detroit Medical Center, Detroit, MI, USA

Received: 10 June 2020

Accepted: 13 July 2020

\section{*Correspondence:}

Dr. Devan O. Higginbotham,

E-mail: higginbothamdevan@gmail.com

Copyright: (C) the author(s), publisher and licensee Medip Academy. This is an open-access article distributed under the terms of the Creative Commons Attribution Non-Commercial License, which permits unrestricted non-commercial use, distribution, and reproduction in any medium, provided the original work is properly cited.

\begin{abstract}
An 11-year-old male sustained an irreducible, completely displaced epiphyseal fracture of the proximal interphalangeal joint (PIPJ) of the middle finger with an associated central slip injury. Central slip injuries occurring in conjunction with Salter-Harris type II middle phalanx fracture are rare entities, with no previously documented case. Our patient underwent open reduction, internal fixation through crossed-pins to achieve reduction and fixation. He was then splinted in extension for six weeks to allow healing of the central slip injury. At 1-year follow-up, the patient had full range-of-motion with no clinical indication of physeal disruption or growth arrest of the repaired digit. We demonstrate a case in which ephiphyseal fractures of the proximal interphalangeal joint with a concomitant central slip injury can safely be treated with open reduction internal fixation combined with a period of immobilization. We characterize a surgical method of reduction and fixation with splinted immobilization and describe lessons learned from this previously unreported case.
\end{abstract}

Keywords: Central slip, Phalanx fracture, Epiphysis, Hand, Trauma

\section{INTRODUCTION}

In the pediatric population, phalangeal fractures represent the most common type of hand fracture, with middle phalanx fractures comprising $16 \%$ of all hand fractures. ${ }^{1}$ Salter-Harris type II fractures are the most common type of finger fracture pattern in the pediatric population. ${ }^{2,3}$ Management of phalangeal fractures is based on the initial severity of injury and depends on the success of closed reduction techniques. ${ }^{3}$ Soft-tissue stability of the proximal interphalangeal (PIP) joint is provided by the ulnar and radial collateral ligaments, volar plate, dorsal capsule, lateral bands, flexor tendon sheath and central slip. ${ }^{4}$ Central slip disruption or laceration of the extensor tendon at the PIP joint with volar displacement of the lateral bands can result in Boutonniere deformity is a deformed position of the fingers or toes, in which the joint nearest the knuckle (the proximal interphalangeal joint, or PIP) is permanently bent toward the palm while the farthest joint (the distal interphalangeal joint, or DIP) is bent back away (PIP flexion with DIP hyperextension), with an extensor lag at the PIP joint. ${ }^{5}$ Central slip injuries occurring in conjunction with Salter-Harris type II middle phalanx fracture are rare entities. Limited published medical literature exists regarding this injury pattern, and we describe an indication for open reduction internal fixation with good outcome regarding range-of-motion (ROM) and clinical satisfaction at 1-year follow-up.

\section{CASE REPORT}

An 11-year-old male was a restrained passenger in a motor vehicle accident. He had immediate right middle finger pain, deformity and bleeding. He initially presented to an outside hospital, where he was found to have a middle phalanx fracture as well as exposed flexor tendon due to a $1 \mathrm{~cm}$ transverse laceration over the PIP crease. He was subsequently transferred to our pediatric 
Level 1 trauma center for management and assessment by a hand surgeon. When evaluated at bedside, the patient was unable to flex the DIP joint and could only move minimally through the PIPJ. He exhibited normal sensation without paresthesias or numbness. Preoperative radiographs were taken, which demonstrated a Salter-Harris II fracture involving the proximal portion of the middle phalanx of the middle finger (Figure 1-3). The middle phalanx shaft was completely dorsally displaced, and the epiphysis was essentially flexed 90-degrees and situated volarly to the proximal phalanx head. There was a small fracture of the dorsal metaphysis, concerning for a central slip injury (Figure 3 ).

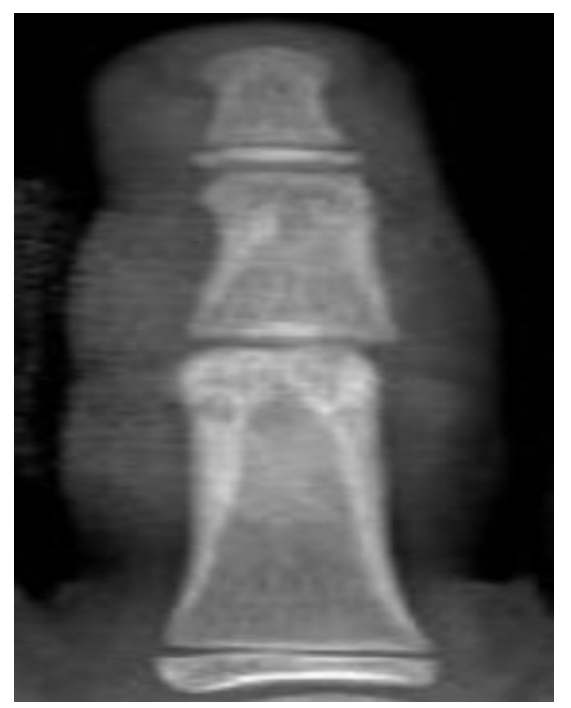

Figure 1: Anteroposterior (AP) radiograph of right middle digit demonstrating a Salter-Harris type II fracture involving the Proximal Interphalangeal (PIP) Joint.

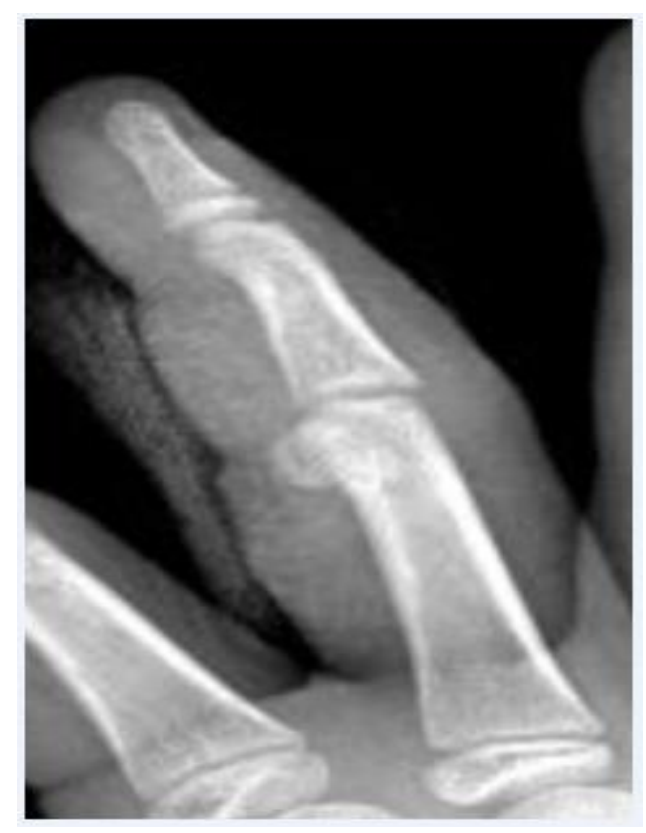

Figure 2: Oblique radiograph of right middle digit.

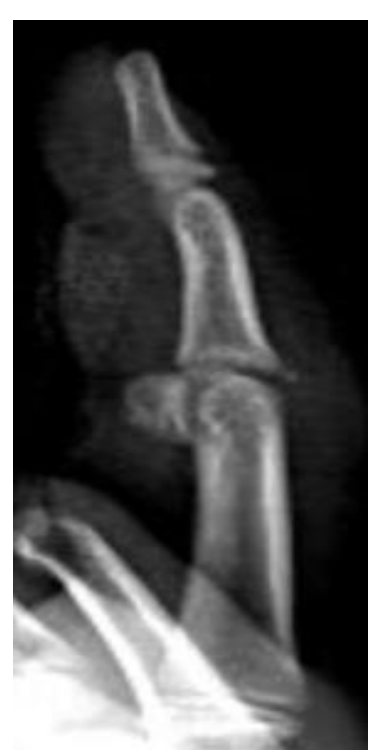

Figure 3: Lateral radiograph of the right middle digit with a dorsal bony avulsion concerning for a central slip injury.

At the time of surgery, an attempt at closed reduction was made using gentle traction to reduce the shaft back over the epiphysis. Fluoroscopy demonstrated incomplete reduction. The volar laceration was extended at the PIP flexion crease on the volar surface to the mid-lateral lines. Mid-lateral incisions were used distal and proximally to extend the exposure. Dissection went down to the flexor tendon sheath and a large disruption of the A3 pulley was noted. The volar plate was avulsed off the middle phalanx. The central slip was difficult to appreciate from the volar approach, but it appeared to be appropriately positioned. Fracture reduction was obtained via traction on the middle and distal phalanges, and once distraction was obtained, the growth plate was gently reduced under the rest of the digit. Using biplanar fluoroscopy, approximately $90 \%$ reduction was visualized, then another period of traction was applied to relax the soft tissues, and the physis was again pushed under the distal part of the finger in order to minimize injury to growth plate. Acceptable reduction was confirmed via biplanar fluoroscopy and direct visualization. The joint and fracture were inspected, and the reduction appeared to be correct.

The reduction was stabilized with 0.028 -inch Kirschner wires from the proximal lateral aspect of the growth plate across the physeal separation, and antegrade into the phalangeal shaft. The K-wires were in appropriate position on both the frontal and lateral views. The volar plate was repaired back to the remainder of the capsule and collateral ligaments, which led to an excellent repair. After all the wounds were closed, the finger was ranged and there was full range of passive motion even with the crossed pins. The pins were trimmed outside the skin and folded over (Figure 4 and 5). The entire finger was placed in an Aluma foam splint to stabilize the fracture in extension to protect the bony central slip injury. The pins 
were removed at 4 weeks. His finger was kept splinted in extension for 6 weeks to ensure healing of the dorsal fracture.

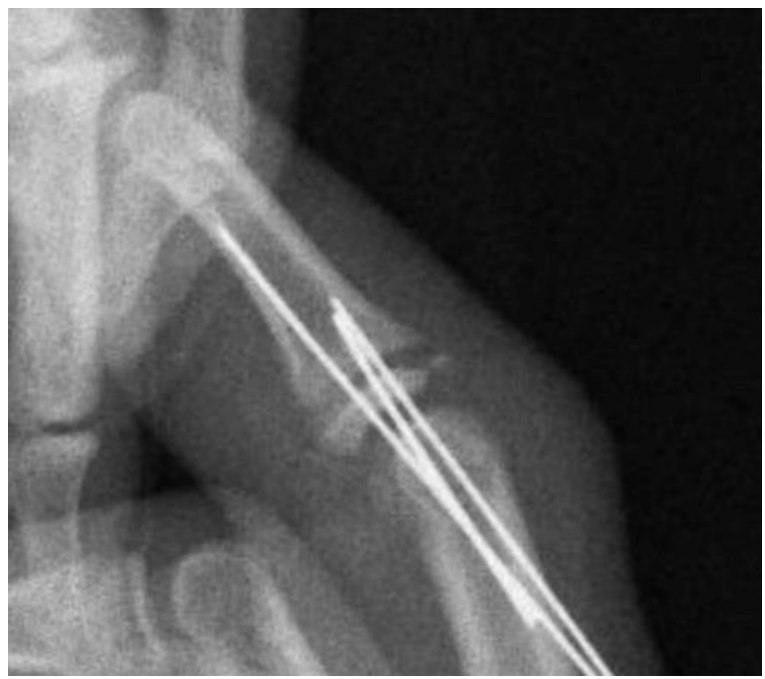

Figure 4: Intra-op radiograph demonstrating reduction with Kirschner-wire stabilization.

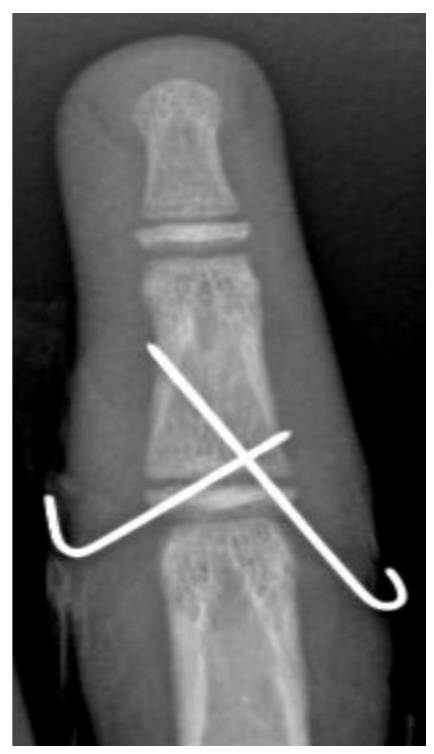

Figure 5: Post-operative image demonstrating successful reduction with crossed-pins.

At two months post-operatively, his x-rays appeared appropriate, and there was evidence of healing of the central slip insertion with bridging across the physis posteriorly (Figures 6 and 7). He was instructed to begin moving his finger independently to regain motion. Three months post-operatively, he was discharged from the clinic as he was regaining motion and demonstrating no other problems. He was instructed to return 1-year postoperatively for $\mathrm{x}$-rays and monitor for growth plate arrest or deformity. The patient was contacted by phone at 1year post-operatively as he did not return to clinic. He was not having further problems with his finger at that time, reported near symmetric ROM, reported complete satisfaction with the outcome, and declined to return for $\mathrm{x}$-rays or clinical exam.

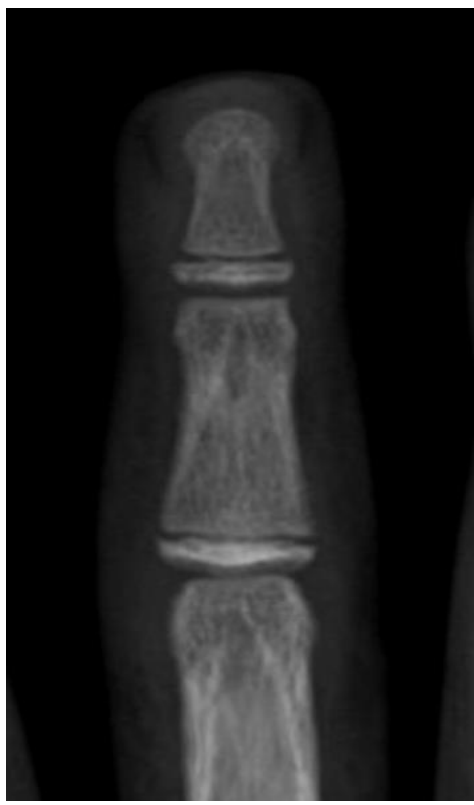

Figure 6: AP radiograph demonstrating acceptable alignment and bridging bone across the physeal disruption 2 months post-operatively.

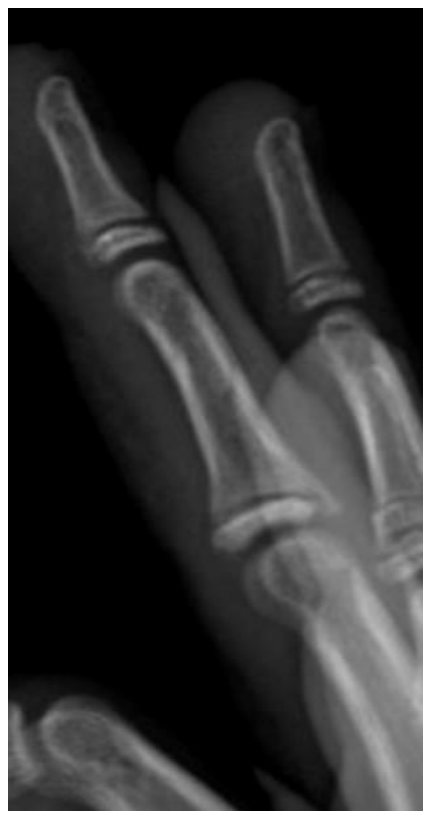

Figure 7: Oblique radiograph demonstrating healed Salter-Harris type II fracture involving the PIP joint 2 months post-operatively.

\section{DISCUSSION}

Middle phalanx fractures are the $3^{\text {rd }}$ most common hand fracture in the pediatric population. ${ }^{1}$ Minimally or nondisplaced fractures are treated with immobilization with expected good outcomes. Displaced injuries like our patient, can lead to deformities which can develop into 
compromised hand function. Our patient had a completely displaced Salter-Harris type II fracture successfully treated with open reduction and internal fixation, with a combination of splinting in extension for 6 weeks for healing of the central slip injury. The literature is limited regarding treatment modalities for a patient with this combination type injury.

Boyer et al concluded closed reduction and percutaneous pinning (CRPP) of displaced, unstable proximal phalanx fractures in children is safe but commonly experience early stiffness. ${ }^{6}$ In their study of 105 proximal phalanx fractures treated with CRPP, they reported patients had full motion with no pain and reported good outcomes at 1-year follow-up, although minor deformity existed in a few. General trends have shown excellent return of grip strength and good function despite some ongoing pain and stiffness. ${ }^{7}$ Al-Qattan examined 34 juxta-epiphyseal fractures, including 16 Salter-Harris type II fractures. ${ }^{8}$ They reported one of their fractures was irreducible due to entrapment of the flexor tendons which was subsequently treated with Kirschner-wire fixation. Previously reported causes of irreducible fractures that are secondary to soft tissue entrapment include flexor tendons, fibrous tissue, and extensor hood and periosteum. ${ }^{8,9}$

Spinner and Choi first documented cases of palmar dislocations of the PIP joint associated with a rupture of the central slip of the extensor mechanism. ${ }^{10}$ In their 5 reported cases, they suggested the injuries were caused by both varus and valgus directed forces combined with a severe anteriorly directed force on the base of the middle phalanx. They recommended operative intervention for reduction with a repair of the central slip. In light of a severe fracture requiring surgical fixation, it was important to not miss a concomitant central slip injury necessitating immobilization but not necessarily repair.

Jones and Jupiter described an irreducible palmar dislocation of the proximal interphalangeal joint associated with an epiphyseal fracture of the middle phalanx. ${ }^{11}$ They reported a case of a palmar dislocation of the PIP joint deemed irreducible due to a displaced epiphyseal fracture. Jones attributed the epiphyseal separation to a consequence of the closed reduction maneuver that was comprised of gentle longitudinal traction with both the metacarpophalangeal and PIP joints flexed. They described the epiphysis rotating 90 degrees and laying with its articular surface facing palmarward preventing the reduction of the dislocation. This was described as a Salter-Harris type I fracture that they describe was mostly due to the patient's spondyloepiphyseal dysplasia making his epiphysis susceptible to a shearing force. However, there was no described central slip injury.

We believe this is the first reported case of an irreducible Salter-Harris type II fracture with volar displacement of the physis with an associated central slip injury. This case highlights important learning points for the treating physician regarding initial, surgical, and post-operative management. First, management of the central slip injury requires splinting because immediate ROM may not allow adequate healing. ${ }^{12}$ The central slip injury could have been overlooked in light of the more severe injury to the epiphysis. The desire to get patients moving to avoid stiffness could have been detrimental to the second and more subtle injury. Digital stiffness, particularly with immobilization for the central slip injury, was discussed with the patient's family at the time of initial evaluation to establish proper expectations. Second, the treating physician must be careful with physeal injuries regarding the PIPJ and closed reduction techniques may be contraindicated. Repeated or over-forceful reduction, either during management in the emergency room or in the operating room, could cause injury to the growth plate in these cases. Despite limited attempts at closed reduction, there was still evidence of bridging bone posteriorly across the growth plate; though at 1 year it had not created a clinical deformity. Lastly, our operative technique is one of many ways that can be utilized. Given the good result our patient experienced after 1-year follow-up, our method is a safe approach in treating this injury.

\section{CONCLUSION}

Salter-Harris type II fractures are the most common phalangeal fracture seen in the paediatric population. This fracture pattern combined with a central slip injury has been previously unreported. Our surgical approach of using crossed-pins in conjunction with immobilization of the digit in extension provided a means for adequate healing of both the fracture and central slip. This case provides an example of how the need to examine for subtle injuries with phalangeal fractures, the importance of use of caution when attempting closed reduction and the use of crossed-pins with immobilization can be utilized for a good outcome at 1-year follow-up.

Funding: No funding sources

Conflict of interest: None declared

Ethical approval: Not required

\section{REFERENCES}

1. Koshy JC, Grome LJ, Schultz K, Bell B, Reece E, Pederson WC. The epidemiology of operative pediatric hand trauma: A retrospective chart review. Hand (N Y) 2019.

2. Abzug JM, Dua K, Bauer AS, Cornwall R, Wyrick TO. Pediatric phalanx fractures. J Am Acad Orthop Surg 2016;24:174-83.

3. Vadivelu R, Dias JJ, Burke FD, Stanton J: Hand injuries in children. J Pediatr Orthop 2006;26(1):2935.

4. Liss FE, Green SM: Capsular injuries of the proximal interphalangeal joint. Hand Clin 1992;8(4):755-68. 
5. Boussakri H, Azarkane M, Dahmani O, Elidrissi M, Shimi M, Elibrahimi A, Elmrini A. Unusual combinations of lesions of the traumatic hand: Closed central slip laceration of the extensor and interphalangeal thumb joint's dislocation (A case report). Pan Afr Med J 2014;18:230.

6. Boyer JS, London DA, Stepan JG, Goldfarb CA. Pediatric proximal phalanx fractures. Outcomes and complications after the surgical treatment of displaced fractures. J Pediatr Orthop 2015;35(3):21923.

7. Hamilton LC. The acute management of unstable intra-articular fractures of the base of the middle phalanx: A systemic review. J Hand Surg Asian Pac 2018;23(4):441-9.

8. Al-Qattan MM. Juxta-epiphyseal fractures at the bof the proximal phalanx of the fingers in children and adolescents. J Hand Surg Br 2002;27(1):24-30.
9. Cornwall R, Ricchetti ET. Pediatric phalanx fractures: Unique challenges and pitfalls. Clin Orthop Relat Res 2006;445:146-56.

10. Spinner M, Choi BY. Anterior dislocation of the proximal interphalangeal joint. J Bone Joint Surg [Am] 1970;52:1329-36.

11. Jones NF, Jupiter JB. Irreducible palmar dislocation of the proximal interphalangeal joint associated with an epiphyseal fracture of the middle phalanx. J Hand Surg Am 1985;10(2):261-4.

12. Pratt AL, Burr N, Grobelaar AO. A prospective review of open central slip laceration repair and rehabilitation. Journal of Hand Surgery (British and European Volume) 2002;27B(6):530-4.

Cite this article as: Higginbotham DO, Tsai AG. Salter-Harris type II fracture of the middle phalanx with concomitant central slip injury in an 11 year old: a case report. Int J Res Orthop 2020;6(5):1130-4. 